\title{
Revista
}

\section{LE SYNDROME DE PINOCCHIO OU LA QUESTION DU} DÉPART

PINOCCHIO'S SYNDROME OR THE QUESTION OF DEPARTURE

\author{
Gilles Garcia ${ }^{1}$
}

\section{COMMUNICATION LORS DE LA JOURNÉE DU 13 AVRIL 2017 : LADITE RADICALISATION ENTRE PRÉVENTION ET PROTECTION. LE TRAVAIL (SOCIAL) AVEC LES MINEURS (IUT PARIS DESCARTES PARIS XVI)}

«Au cours de la conversation, abstiens-toi de t'étendre sur tes actions passées, sur les risques que tu as pris : car s'il t'est doux de te remémorer les dangers que tu as courus, le récit de tes aventures n'a pas les mêmes charmes pour les autres. Évite également de faire rire : car non seulement cela peut facilement tomber dans la vulgarité, mais cela risque, en plus, de faire abandonner à tes interlocuteurs leur retenue envers toi. Un autre terrain glissant, c'est quand on en vient à parler de choses obscènes. Quand cela se produit, si c'est possible, n'hésite pas à reprendre celui qui a commencé. Sinon, exprime au moins clairement, par ton silence, ta rougeur et ton air réprobateur, que cette conversation te déplaît. Quand il te vient l'envie d'un plaisir, comme pour les autres sortes de représentations, prends garde de ne pas céder à sa violence : laisse reposer la chose et accorde-toi un délai ; songe à ces deux instants : celui où tu goûteras le plaisir et celui où, après y avoir goûté, tu en auras le regret et tu t'insulteras toi-même tout bas. Oppose à cela la joie que tu éprouveras et les louanges que tu t'adresseras si tu t'abstiens. Si tu trouves opportun de passer à l'acte, fais attention de ne pas succomber à la douceur agréable et séduisante de la chose. Image, pour y résister, combien précieuse est la conscience d'avoir remporté cette victoire-là »[1].

II ne s'agit pas ici de traiter du passage à l'acte violent chez l'adolescent à partir du phénomène de ladite radicalisation, mais j'essaierai d'interroger les mécanismes à l'œuvre et en puissance contribuant au départ psychique et/ou réel de «nos jeunes » français. Le contexte politique choisi pourrait se condenser dans La France tu l'aimes ou

\footnotetext{
1 Formateur-chercheur et psychanalyste, Ecole Supérieure de Travail Social Paris - Sorbonne, Paris IV. - ER : Rationalités Contemporaines, 3 villa des Bruyères 94800 Villejuif. France. E-mail : gilles.garcia@etsup.com
} 
tu la quittes !, slogan en vogue lors de la création du ministère de l'Immigration, de l'Intégration, de l'Identité nationale et du Développement solidaire. On remarque déjà les effets possiblement ravageurs d'un appel ambigu à l'amour corrélé avec une représentation de l'identité. "Qui suis-je ? Rien, personne. La conviction vécue d'être dans une impasse subjectale est la prédisposition la plus forte pour l'acte de partir et devenir ainsi la proie des recruteurs »[2]. Cela sans oublier l'effet de rejet conjuguée avec la violence, naturellement adolescente, rappelons que les forces de la jeunesse - Epictète nous rappelle qu'il n'est pas toujours aisé de modérer la surexcitation sexuelle - et les forces armées sont les premières occurrences de l'origine latine de la violence, vis.

Nos jeunes, dans la mesure où nous avons choisi de considérer leur départ comme un phénomène qui mérite notre attention dans leur façon de vivre leur vie pour qu'elle en vaille la peine. Une vie avant la mort ! L'effet de loupe des récents attentats permet de jeter un regard en arrière et dans l'après-coup sur quelques signes avant-coureurs. Partir pour où ? Pour le combat (hijra) : Syrie... Pour ma vraie patrie (alya) : Israël... Ceci est un point de départ et de rupture qui ne relève pas que du registre de l'émotion et de la désinformation. De même que le complot interroge la dimension paranoïagène du rapport à l'Autre, cet amour de la patrie fait appel au père.

Pour le titre : CEil de pin, bois de chauffe doué de la parole, nœud dans le bois, Pinocchio! C'est d'abord une " course-pour-la-mort » qui constitue la première partie du conte tragique de Collodi [3] qui utilise tous les thèmes initiatiques que nous connaissons bien. Toutefois, il y a la question de la séduction des liens adulte/enfant et d'une forme de fraternité dans l'ânerie qui nous intéresse particulièrement chez ces jeunes qui partent, que le voyage soit réel ou virtuel, soit une dimension structurale propre à l'adolescence de quitter un corps, un lieu, un idéal. Mon deuxième terme étant le fait que le syndrome porte lourdement sur l'enfant à la différence d'un symptôme qui est plutôt celui de la famille, il s'agit donc d'interroger cette place paternelle qui fonctionne ou pas, sur un temps comme idéal, nous y reviendrons, sans compter l'institution politique. Comment accueillir et retenir le départ, accompagner le choix de vie et faire supporter le tiers? Le mythe freudien du père pris en défaut dans cette configuration impliquerait de s'inventer une normalisation supportable et à l'abri du désir de l'Autre. Cette normalisation correspond à une autorisation mais elle peut aussi être vécue comme un écrasement voire une exclusion réelle. L'agir produit par un désir affranchi joue contre l'angoisse d'exclusion qui se condense dans le ressentiment de ne pas avoir de place. «Le désir existe, est constitué, se promène à travers le monde, et il exerce ses ravages avant toute tentative de vos imaginations, érotiques ou pas, pour le réaliser, et même, il n'est pas exclu que vous le rencontriez comme tel, le désir de l'Autre, de l'Autre réel tel que je l'ai défini tout à l'heure. C'est en ce point que naît l'angoisse, c'est la sensation du désir de l'Autre »[4]. C'est le choix forcé de Charybde et Scylla, ou rejeté ou englouti par le gouffre de l'Autre.

Alors le départ de Pinocchio est-ce une fugue de l'angoisse de castration? non dans la mesure où n'étant pas marqué par la loi du Père, tout est permis, sauf qu'il apprend à ses dépens que justement si Dieu est mort rien n'est permis. La réalité se charge de lui montrer de quel bois elle se chauffe. Pantin fabriqué, prototype d'une chaine d'enfants soldats qui n'ont de salut que parce que leur parole est possible, c'est la parole dans le bois de pin, si on ne l'entend pas elle part au feu... Souvent on se débarrasse des erreurs or le problème freudien réside en partie dans le fait que le sujet se trouve plus dans l'erreur et le ratage que dans la réussite. Et pour cause, quand l'acte est réussi, le sujet a disparu dans le suicide. La force de Pinocchio tient dans ce qu'il n'est pas dans la demande de l'Autre : «Che vuoi ? ", Que me veux-tu ? autrement dit, dans le désir sans la loi. Tant que je désire, je ne sais rien de ce que je désire. En outre, cette dimension de méconnaissance courageuse se paye, Pinocchio l'affranchi meurt d'être sans secours et sans recours. Le chat et le renard le pendent tandis que la petite fille à la fenêtre est un fantôme impuissant 
pour le secourir. Cette jeune fille morte deviendra dans la deuxième partie, la fée bleue.

Qu'est-ce à dire ? Geppetto est sourd ? pas plus que cela puisqu'il n'est pas père, sauf que le conte le fait advenir comme tel, dans l'ultime péripétie du retournement de l'inanimé en animé, en garçon de chair et d'os, mortel et affilié. Son désir de parole, au commencement, s'est incarné, et ils peuvent sortir tous deux du ventre la baleine, bras dessus bras dessous, tel Abraham et Isaac descendant de la montagne de l'Eternel, le pire ayant été évité. Ce n'est pas l'adulte secourable mais tout autant l'enfant secourable : Est-ce un « tu es mon père » ou un « je suis ton fils » qui opère ici?

Ces différentes références pour aborder cette première question du jeune pris dans cette jeunesse qui ne passe pas et dont les voyages, paraît-il, pourraient la former. Est-ce que l'ennui le congédie à l'inanimé avec comme seule alternative l'envie de partir dans un désir de s'enflammer enfin pour quelque chose. Le départ pour la fête au pays de cocagne constitue un véritable divertissement, à savoir une fuite. Comment l'ennui peut-il vaincre l'inhibition ? Nous allons voir que la place laissée, imposée ou automatique voire idéalisée de l'Autre nous aide à prendre en compte ce jeune sujet. En effet, l'inhibition intellectuelle vient marquer la confrontation à l'impuissance de réaliser la demande de l'Autre (à la fois ce qu'il rêve de faire et ce que l'on exige de lui - dimension torique du sujet et l'Autre [), celui qui s'en affranchit, se libère du même coup pour agir, soit pour notre question du jour pour partir. Tandis que l'acting-out est une forme de monstration dont l'agir est adressé à l'Autre tout en restant bloqué sur une scène, le passage à l'acte en est souvent la sortie définitive. Nous reprendrons ces trois termes plus haut loin.

S'ennuyer - inodialre-, en lien donc avec la haine mais de qui et de quoi ? Une haine de soi-même comme son propre objet. Cela pour pointer son importance, encore faut-il l'extraire de l'habitude et du banal, pour en faire un trait voire un symptôme[5]. « On nous pousse à consommer, à consommer, ça ne donne pas une raison de vivre. Certains ont besoin d'un autre projet (à entendre ici la question du désir). Consommer, ça génère de l'ennui aussi, on dirait qu'on est déjà mort (...) Je ressentais un manque, j'avais un vide spirituel à combler et je l'ai comblé avec la religion ». Ce vocabulaire est à prendre au sérieux, c'est-à-dire au pied de la lettre : la perte, le manque et le comblage nous permettent de l'articuler à l'angoisse. Déjà l'angoisse de choisir entre deux opiums : la consommation et la religion, mais surtout une position de déjà-mort. L'angoisse agirait comme signal d'une réapparition fantomatique du a (scybale, mamelon, regard, phallus), en tant qu'il recouvre le champ des objets d'échanges: cotables, socialisés, communicables, concurrentiels (rivalité/accord). Ce qui nous permet d'introduire la formule d'Hamlet confronté à son spectre de père déjà-mort : «It will not speak, I will follow it ».

Ce qui intéresse ici ce n'est la position du père déclinant ou humilié, mais celle du père spectral, en difficulté pour transmettre un désir, faute de vividité, sans que cela soit un impératif catégorique : Che vuoi? Evidemment, cela se complique lorsque le fils déjà-mort remeurt dans le rêve : «Père ne vois-tu pas, je brûle[r] ! » soit la formule traumatique - côté fils $\square$ qui illustre la rencontre manquée. Si rencontre il n'y a pas, il ne reste que l'extérieur pour arrêter cette course-vers-la-mort, à savoir une rencontre de la Loi, à condition qu'elle soit elle-même incarnée, un tiers qui parle...

Dis Syrie ?/ Dis SIRI ? Est une application qui permet de "parler" à son téléphone. Cette homonymie permet d'insister sur le déterminisme objectal qui opère lorsque «le réel commande plus que tout autre nos activités »[8]. Le réel est évidemment de l'autre côté du miroir, au-delà du rêve, cet agir inconscient qui déplace et condense, cache pour mieux montrer, « derrière le manque de la représentation dont il n'y a que le tenant-lieu de la représentation »[9]. Est-ce du fait d'un objet ou d'une machine qui répond dans cette enclave virtuelle qu'il faille pour en sortir engager tout 
son corps? Dans le passage à l'acte, l'hallucination crève l'écran du fantasme, et le sujet tel Lord Jim passe par-dessus bord. Mais le passage en Syrie, marque une autre dimension car il faut être déjà mort pour profiter du temps qui reste. Ce n'est pas une livre de chair séparée du corps pour payer sa dette symbolique, mais tout le corps comme preuve qui légitime l'exaction, doublé d'un achat des péchés sans falsification.

Nous faisons également l'hypothèse que le moment de partir est l'équivalent d'un moment de conclure qui vient consolider ce que nous considérons comme une destruction. Ce vocable fait également écho à la destruction d'emplois provoquée par la « crise » socio-économique et culturelle que l'Occident traverse, nous suivons ici le rapprochement pertinent de Judith Butler[10] entre la mondialisation et les corps. "Les formes contemporaines de l'abandon économique produisent de la précarité, une forme "d'invivabilité"[11] puisque rien ne m'assiste ! Pas digne de m'assister. Comment peut-on se demander quelle est la meilleure vie à mener quand on sent qu'on a aucun pouvoir à diriger sa vie »[12]. Pourquoi survivre pour vivre ? Sur notre rive nous perdons des jeunes, faute de les accompagner à diriger leur vie dans l'illusion que nous avons de diriger les nôtres. Ce n'est pas tant quelle planète nous voulons pour nos enfants, mais quels enfants nous voulons? Autrement dit, la réflexion sur l'éducation - métier impossible - est première sur le dressage et le changement des habitudes écologiques.

D'où vient ce moment de conclure ? il est le dernier des trois temps logiques[13], à savoir l'instant de voir, le temps pour comprendre et le moment de conclure. Par exemple dans le champ de la protection de l'enfance, l'expérience peut nous montrer qu'à signaler trop promptement, soit à conclure qu'il faut signaler dans l'intérêt de l'enfant, on puisse se rendre compte dans l'après-coup que l'on a jeté le bébé avec l'eau du bain. Ce qui constitue un écrasement du temps pour comprendre[14], moment utile pour résister à l'automatisme de l'acte.

Partir de chez-soi constitue un déplacement du Heim - je n'ai pas ma place ici, elle est ailleurs ! - vient découper un chez-soi antérieur. Si j'y suis là-bas c'est au moins la double conséquence d'une exclusion que nous dirons pour le moment imaginaire et d'un désir puissant d'en découdre avec l'étranger. " II nous faut admettre, dit Serge Tisseron, qu'il y a quelque chose d'éternel dans leur révolte, mais s'il existe dans des conditions objectives radicales nouvelles "[15]... Pour interroger cet autre de la vie qui est ailleurs il ne suffit pas de marcher sur les traces rimbaldiennes qui ont déjà traversé la méditerranée, mais aussi de reprendre la clinique adolescente qui fait du domicile subjectif un lieu brûlant et passionné. Pour Philippe Gutton « «faire le djihad » est un symptôme de la situation adolescente, au même titre que la tentative de suicide, l'automutilation, l'addiction, la fugue... (...) l'adolescent y est d'une sensibilité extrême (au point que j'ai parlé d'une paranoïa ordinaire même nécessaire) »[16], soit une attaque du corps propre commandée par une idée forte, fixe, noire et impérative.

D'où vient l'appel sauvage ? de l'intérieur ou de l'extérieur? Autrement dit est-ce un père qui appelle certains jeunes à quitter leur lieu quand il ne les dévore pas, si tant est que ce dernier ait été suffisamment bon et accueillant pour que ce lieu fonctionne comme un chez-soi. Le contexte hétérocide actuel du fait religieux permet également d'interroger la structure de ces noms-du-père qui les rallient sous leurs bannières. « II y a autre chose à entendre de l'ordre angoissant de Dieu, il y a la chasse de Diane, il y a l'hallali du loup »[17].

Point de conclure ou de départ, point d'acte et de rupture aussi, sachant que les signes de rupture constituent les symptômes avancés d'une emprise mentale et d'une dite radicalisation. La problématique sectaire connaît bien cet autodafé qui laisse brûler en premier non pas les livres et les papiers identité mais l'album de famille. Cette déconnaissance à l'égard des siens est-elle l'épiphénomène d'un échec parental ou bien la marque d'un acte réussi d'en être enfin... et ce pour un temps relativement court? MV Bill disait que l'espérance de vie est de11 ans pour les 
faucons, pour les djihadistes, un peu plus quand ils ne sont pas bombardés par la France! Attention c'est facile de dire cela si nous ne travaillons pas la question de l'amour filial. « Marie-Jean Sauret insiste sur notre incapacité à penser l'altérité autrement que comme un danger à combattre : " Si nous ne sommes pas capables d'accueillir convenablement l'altérité là où elle se voit, il n'est pas étonnant qu'elle soit maltraitée là où elle est moins soupçonnée $»[18]$.

Nous parlions d'un départ réel ou virtuel, du coup notre question sur les contenus de cette " agencéité », cette capacité à agir présente dans la conviction et l'intention tandis que sur l'autre versant gît l'acte. Nous savons que " quand tu pourras, tu feras » relève de la promesse œdipienne qui de structure est non tenue mais autorise à aller voir ailleurs. Pourquoi dans cet espace intime, un autre vient faire croire qu'il tiendra cette promesse, n'est-ce pas un père imaginaire qui réalise un vœu ? en outre, la réalisation de cette promesse ne semble plus relever du désir mais de la jouissance. Pourquoi ? Car l'objet a-regard est mis en scène dans une monstration que l'on appelle acting-out. Ainsi, la question poursuivie est la suivante, dans ces affaires, ne s'agit-il pas d'un acting out plutôt que d'un passage à l'acte ? autrement dit, il se passe quelque chose sur la scène qui fait référence à une autre scène... la scène du fantasme qui se joue entre le désir du sujet et le désir de l'Autre. "Qui est celui qui nous donne d'abord l'exemple de la castration attirée, assumée, désirée comme telle, sinon CEdipe ? OEdipe n'est pas d'abord le père, c'est ce que j'ai voulu dire depuis longtemps en faisant remarquer ironiquement qu'CEdipe n'aurait su avoir un complexe d'CEdipe. Le péché d'CEdipe est la cupido sciendi, il veut savoir. Et ceci se paie par l'horreur que j'ai décrite que ce qu'il voit enfin, ce sont ses propres yeux, a jetés au sol »[19].

Les yeux de pin, crevés, arrachés[20] précipite la prévalence de la dimension scopique, les écrans jouant le rôle ambivalent de faire à la fois écran et miroir, c'est une question de tain ? il se montre-il est vu-il voit, c'est une séquence que l'on peut difficilement séparer. Prenons comme exemple les jeunes "sur zone » qui se montre dans des lieux premium ou de cocagne, avec cette remarque importante, sans compter, les insupportables que cela peut renvoyer : à savoir il déplace leur " culture de cité » dans les hôtels de Syrie : autrement dit c'est vraiment des « racailles »; leur sourire[21] - drogué ou pas - semble relever d'une jouissance qui est adressée au spectateur: comment peuvent-ils ? ; mais côté verbatim : qu'est-ce que les jeunes disent ? ici Zoubeir : " par moments, c'était troublant. On n'avait plus l'impression de se retrouver dans un pays en guerre. On avait l'impression d'être en train de s'amuser, comme si on était parti à la plage, en vacances. C'est bien le terme "LOL jihad (importation en Syrie d'« une culture des cités »)", c'était bien ça (...) Un jihad cinq étoiles. Un jihad où on peut tirer sur les gens et manger une glace en même temps »[22].

Cet extrait des Revenants - que l'on peut aussi entendre comme le retour des morts qu'il faut réintégrer, si tant est qu'ils aient une place $\square$, il est certes possible d'insister sur cette dimension de carnaval, de retournement des valeurs où ce qui n'était pas possible devient permis. Mais il était également possible de demander où sont les parents ? $\mathrm{Ce}$ n'est pas une position réactionnaire qui les culpabiliserait ou les responsabiliserait. Mais c'est un retour à la scène sexuel du fantasme dans son versant tragique : «Père ne vois-tu pas je brûle? ».

Le sentiment de culpabilité inconscient devient, à partir de 1924[23], le besoin de punition de la part d'une puissance parentale. Du même coup, l'identification narcissique supplante le complexe d'CEdipe, ce sont les Imagines (référence à l'image) qui l'emportent sur l'acoustique et l'entendu. Car le besoin de punition met en relation l'autopunition liée à la pulsion de mort et la satisfaction libidinale liée à la pulsion sexuelle. En effet, comme le masochisme moral a « la signification d'une composante érotique, même l'autodestruction de la personne ne peut se 
produ vbcire sans satisfaction libidinale "[25]. Freud reprend ici une déduction produite par la reconstruction du fantasme dans un de ses textes majeurs, Un enfant est battu, car cet «être-battu »[26] se fonde d'un je suis aimé par le père. Qu'est-ce qui motive donc une telle décharge haineuse et agressive ? La pulsion de mort - retour vers l'inanimé $\square$ cause cette haine du surmoi[27] du fait de l'identification avec le modèle paternel, identification sur le mode d'une désexuation provoquée par la désintrication[28] pulsionnelle. Ainsi, le résidu érotique issu du " démélange » qui reste libre après l'éclatement serait à l'origine de cette tendance impérative voire agressive de l'idéal du moi (surmoi). Cette désexuation ne signifie pas que le sexe a disparu, il origine cette violence en étant transformé.

«Eh bien! quand nous en serons arrivés la - vous pouvez quand même déjà le voir indiqué dans mille références, les références à Sade, pour prendre les plus proches », le fantasme un enfant est battu / un homme est décapité... cela ne réduit pas à des associations dans le mesure où il s'agit de s'intéresser non pas à ceux qui produisent sciemment une barbarie, mais à ceux qui les regardent afin de s'interroger sur le statut de ce qu'il voit: est-ce de l'ordre du fantasme ? a qui s'identifie-t-on ? au corps à découper ou au bourreau ? La théorie de la pensée inconsciente du rêve nous apprend que nous sommes tous les sujets à la fois, qu'il n'y a pas de « je suis » mais du " ça pense ».

Nous allons voir à présent à travers quelques éléments de cas célèbres de Freud certaines formes de conversion pas nécessairement hystériques. Deux événements majeurs ont déterminé l'entrée en analyse de la jeune homosexuelle (FREUD S., De la psychogenèse d'un cas d'homosexualité féminine, in Névrose, psychose et perversion, Paris, PUF, 1973), ils condensent son histoire de façon économique. À savoir le caractère de monstration de la jeune fille lorsqu'elle s'affiche en public avec la demi-mondaine, et la tentative de suicide prise comme une preuve univoque d'amour (qui constitue le passage à l'acte). On ira de ses sentiments aux regards (on s'efforcera d'insister sur l'importance du regard dans ce cas) de ses parents et de la Dame jusqu'à l'issue qui est la « solution » de ce conflit insupportable.

Grâce à la présentation des faits que propose Freud, on peut envisager au moins deux niveaux d'interprétation. Le premier niveau s'appuie sur deux faits de surface qui ont évidemment leur importance mais ne se suffisent pas à eux-mêmes : En se promenant embrassée avec son amie près des bureaux de son père, la jeune fille croise ce dernier qui, sans un mot, lui lance un regard courroucé. La Dame, interdite, lui demande qui est ce monsieur qu'elles viennent de rencontrer. La jeune fille, presque désinvolte, lui répond que c'est son père et la bien-aimée se met alors en colère et lui intime de rompre sur-le-champ : «—Dans ces conditions, on ne se revoit plus ». L'objet regard du père et de la demi-mondaine est à présent formulé, venant mettre des mots sur ce qui n'en avait pas. Prise de désespoir, la jeune fille saute le parapet d'un pont qui surplombe une voie ferrée. Par chance, aucun train ne passait à ce moment-là ! Mais le désespoir est-il une cause suffisante?

Le second niveau d'interprétation, analytique cette fois, permettra d'examiner l'instant d'avant l'acte de folie de la jeune fille. Pour ce faire, on introduira des éléments de son anamnèse afin de la présenter à rebours. De prime abord Freud fait remarquer que sur le plan symbolique la jeune fille identifie les deux scènes exactement de la même manière. II y a un recouvrement pour le sujet des deux interdictions, voire une confusion hallucinatoire. Si l'on utilise les coordonnées du tableau, on peut se demander quel empêchement ajouté à l'émoi provoque chez la jeune-fille l' acting-out, cette mise en scène dans les rues de Vienne et plus particulièrement sous les fenêtres de son père ? Tandis que les coordonnées de l'angoisse permettent de définir les conditions essentielles du passage à l'acte. En effet, au suprême embarras causé par le regard du père s'ajoute l'émotion due à la subite impossibilité de faire face à 
la scène que lui fait son amie, ce qui a pour conséquence un passage à l'acte. Lacan définit cet agir par une identification absolue à l'objet petit a auquel la jeune fille se réduit. « Personne ne trouve peut-être l'énergie psychique pour se tuer si, premièrement du même coup, il ne tue pas ainsi du même coup un objet avec lequel il s'est identifié et si, deuxièmement, il ne retourne pas par là contre soi-même un souhait de mort qui était dirigé contre une autre personne $»[22]$.

L'axe de la difficulté[30] serait celui du sujet et du désir tandis que celui du mouvement serait celui du moi.

Différence inhibition et angoisse : apparition variable de l'objet $a$; ces deux mécanismes sont aux deux bouts de la chaîne (diagonale) :

La tentative de suicide recouvre donc de façon condensée plusieurs réalisations: un accomplissement d'autopunition mêlé à l'accomplissement d'un souhait (mettre bas : niederkommen). La patiente met en acte inconscient le jeu de mots au lieu de le formuler : elle se laisse tomber, niederkommen. L'analogie avec l'accouchement est loin d'épuiser son sens. «Ce niederkommen est essentiel à toute subite mise en rapport du sujet avec ce qu'il est comme a. Ce n'est pas pour rien que le sujet mélancolique a une propension telle, est toujours accomplie avec une rapidité fulgurante, déconcertante, à se balancer par la fenêtre »[31].

Au point où j'en suis et je terminerai là-dessus, car ce qui pourrait s'apparenter à un passage à l'acte violent inauguré par le départ semble se rapprocher d'une conversion, qui, comme nous le rappelle Pierre Hadot (ça ne s'invente pas) correspond à un «retournement, changement de direction, dans son acception religieuse et philosophique ; il s'agira alors d'un changement d'ordre mental, qui pourra aller de la simple modification d'une opinion jusqu'à la transformation totale de la personnalité. Le mot latin conversio correspond en fait à deux mots grecs de sens différents, d'une part epistrophê qui signifie changement d'orientation et implique l'idée d'un retour (retour à l'origine, retour à soi), d'autre part metanoïa qui signifie changement de pensée, repentir, et implique l'idée d'une mutation et d'une renaissance. II y a donc, dans la notion de conversion, une opposition interne entre l'idée de " retour à l'origine » et l'idée de « renaissance »[32].

Cette extension de la conversion tient également de l'acting-out et du passage à l'acte même s'il n'est pas vécu comme tel. Car "chacun, dans " l'événement tragique », en ce " grand instant » (Lukács), est nouveau-né et mort depuis longtemps ; c'est sa vie face au jugement dernier [...] Cela se passe toujours d'un coup, soudainement - les préliminaires ne sont là que pour les spectateurs : c'est une préparation de leur âme au saut de la grande conversion » (255) »[33]. Citation précieuse qui permet de situer ce regard convergent d'Internet dans le moment moderne de la conversion dont les coordonnées logique et tragique du retournement. La conversion porte en elle-même l'ambiguïté du retour et de la mutation. Soit une autre forme résolutive de la question adolescente car c'est dans la mesure où ce qu'il s'agit d'éviter, c'est ce qui, dans l'angoisse, se tient d'affreuse certitude or c'est justement de l'angoisse que l'action emprunte sa certitude : Agir, c'est arracher à l'angoisse sa certitude. Agir, c'est opérer un transfert d'angoisse.

\section{Références bibliographiques}

Butler, J. (2014), Qu'est-ce qu’une vie bonne ? Paris, France : Payot \& Rivages, 2014, p.73. 
Darriulat, J. $(2007=, 29$ octobre). Introduction à la philosophie esthétique. consulté sur : http://www.jdarriulat.net/Introductionphiloesth/Antiquite/Tragediephilo/SituaTragique1.html

Epictète Manuel, XXXIV, trad. Pépin p. 1126, que l'on souligne.

Garcia, G. (2012, 10 juin). RAS (Rien à Signaler) ? entre responsabilité et évaluation. Consulté sur : http://www.psychasoc.com/Textes/RAS-Rien-a-Signaler-Entre-responsabilite-et-evaluation.

Gutton, P. (2016, avril-mai-juin). « Partir » en adolescence, L'école des parents, sup. au 619(5), 197-204. Adolescents en quête de sens : parents et professionnels face aux engagements radicaux,

Hadot P., Exercices spirituels et philosophie antique, Paris, Albin Michel, octobre 2002, pp. 226-227.

Raynaud, J. (2016, avril-mai-juin). Pinocchio, ou le risque de la radicalisation à l'adolescence. L'école des parents, sup. au $619,99-123$.

Thompson, D. (2016). Les revenants : Ils étaient partis faire le jihad, ils sont de retour en France, Paris, France : Seuil.

Tisseron, S. (2016, avril-mai-juin). Des leviers pour développer l'empathie et prévenir les dérives radicales. L'école des parents, sup. au 619, 125-143.

\section{NOTAS}

\footnotetext{
Notas

11 ÉPICTETE Manuel, XXXIV, trad. Pépin p. 1126, que l’on souligne.

${ }^{2} 2$ GUTTON P., « Partir » en adolescence, p. 202 in Adolescents en quête de sens : parents et professionnels face aux engagements radicaux, sous la direction de MARCELLI

D., Eres, 2016, coll. EPE.

${ }_{3}^{3} 3$ COLLODI C., Les Aventures de Pinocchio : histoire d'un pantin, Jean-Michel Gardair (Sous la direction de), Nathalie Castagné (Traduction), Paris, Folio classique - 10 mars 2003

${ }_{4}^{4}$ LACAN J., Séminaire IX : L’'dentification, leçon 02 mai 1962, p. 415 (cf. http://www.valas.fr/IMG/pdf/S9_identification.pdf)

${ }^{5} 5$ « ... mais qui eût pu discerner le symptôme avant le crime ? » (LACAN J., De la psychose paranoïaque dans ses rapports avec la personnalité, p. 299).

${ }_{6}^{6}$ THOMPSON D., 2016, Les Revenants : Ils étaient partis faire le jihad, ils sont de retour en France, Paris, France : Seuil, p.97.

${ }^{7} 7$ LACAN J., Le Séminaire Livre XI : Les Quatre concepts fondamentaux de la psychanalyse, p. 66. - Vater, siehst du denn nicht, dass ich verbrenne ? Qu'est-ce qui nous réveille tel le père dans son rêve insoutenable ? L'autre réalité cachée derrière le manque de ce qui tient lieu de représentation, le Trieb. « Ainsi la rencontre, toujours manquée est passée entre le rêve et le réveil, entre celui qui dort toujours et dont nous ne saurons pas le rêve et celui qui n’a rêvé que pour ne pas se réveiller » (Le Séminaire Livre XI Les Quatre concepts fondamentaux de la psychanalyse, p. 68).
} 
${ }^{8} 8$ LACAN J., Le Séminaire Livre XI : Les Quatre concepts fondamentaux de la psychanalyse, p. 71.

${ }^{9} 9$ LACAN J., Le Séminaire Livre XI : Les Quatre concepts fondamentaux de la psychanalyse, p. 71.

1010 BUTLER J., Qu'est-ce qu'une vie bonne ?, p. 73.

1111 BUTLER J., Qu'est-ce qu'une vie bonne ?, p. 72. "A la différence logique d'une vie vivable qui puisse être vécue", ibidem, p. 89.

1212 BUTLER J., Qu'est-ce qu'une vie bonne ?, p. 73

1313 Cf. LACAN J., Les trois temps logiques, le syllogisme de l'assertion de certitude anticipée in Ecrits, Paris, Seuil, 1966.

1414 GARCIA G., RAS (Rien à Signaler) ? entre responsabilité et évaluation, in http://www.psychasoc.com/Textes/RAS-Rien-a-Signaler-Entre-responsabilite-et-evaluation.

15 TISSERON S., Des leviers pour développer l'empathie et prévenir..., in Adolescents en quête de sens : parents et professionnels face aux engagements radicaux, sous la direction de MARCELLI D., Eres, coll. EPE.p. 127.

16 GUTTON P., « Partir » en adolescence, p. 197, idem, ibidem.

1717 LACAN J., Séminaire X : L’Angoisse, leçon du 14 décembre 1962, p.133 (cf. http://www.valas.fr/IMG/pdf/S10_L_ANGOISSE.pdf), que l'on souligne.

18 RAYNAUD, Pinocchio, ou le risque de la radicalisation à l'adolescence ; in Adolescents en quête de sens : parents et professionnels face aux engagements radicaux, sous la direction de MARCELLI D., Eres, coll. EPE.2016, p.116

1919 LACAN J., Séminaire X : L'Angoisse, leçon du 3 juillet 1963, p.611 (cf. http://www.valas.fr/IMG/pdf/S10 L ANGOISSE.pdf). Est-ce à dire que ce soit la structure du quatrième niveau et que, quelque part, il y ait toujours présent ce rite sanglant d'aveuglement ? Non. II n'est pas nécessaire - et c'est bien là par quoi le drame humain n'est pas tragédie, mais comédie, ils ont des yeux pour ne point voir, - il n'est pas nécessaire qu'ils se les arrachent. L'angoisse est suffisamment repoussée, méconnue dans la seule capture de l'image spéculaire, i(a), dont le mieux qu'on pourrait souhaiter est qu'elle se reflète dans les yeux de l'Autre. Mais ce n'est même pas besoin, puisqu'il y a le miroir. «Elles arrachent les yeux comme châtraient les Bacchantes. La curiosité sacrilège qui fait l'angoisse de l'homme depuis le fond des âges, c'est elle qui les anime quand elles désirent leurs victimes, quand elles traquent dans leurs blessures béantes ce que Christine plus tard devant le juge, devait appeler dans son innocence, "le mystère de la vie" » (LACAN J., Motifs du crime paranoïaque : Le crime des sœurs Papin, in De la psychose paranoïaque dans ses rapports avec la personnalité, p 399).

${ }^{20} 20 \mathrm{Cf}$. Le supplice du rat, que le patient de Freud racontait avec un sourire de jouissance insue de lui-même.

2121 THOMPSON D., 2016, Les Revenants : Ils étaient partis faire le jihad, ils sont de retour en France, Paris, France : Seuil, p. 123-124.

22 Cf. S. FREUD, Le Problème économique du masochisme Névrose, psychose et perversion, Paris, PUF, 1973.

${ }^{23} 23$ FREUD S., Le Problème économique du masochisme, Névrose, psychose et perversion, Paris, PUF, 1973, p. 297.

${ }^{24} 24$ FREUD S., CEuvres Complètes : psychanalyse, XV, Un enfant est battu, p. 130

${ }^{25} 25$ Le Sur-Moi est conçu par Freud comme une réincorporation d'une partie du monde extérieur, tandis que le Moi n'est que « la surface » du Es.

${ }^{26} 26$ Die Entmischung : (démélange) : gâteau avec tout le sucre d'un côté et le sel de l'autre (Le Problème économique du masochisme, in Névrose, psychose et perversion Paris, PUF, 1973, p. 294).

2727 FREUD S., Le Problème économique du masochisme, in Névrose, psychose et perversion, Paris, PUF, 1973, p. 252.

${ }^{28} 28$ RICOEUR J.-P., (1976 juillet) Inhibition intellectuelle : je pense donc je vaux, in Inhibition et acting-out, Lettres de l'école freudienne, $n^{\circ} 19$, p. 80

${ }^{29} 29$ LACAN J., Séminaire X : L’Angoisse, leçon du 16 janvier 1963, p. 174 (cf. http://www.valas.fr/IMG/pdf/S10_L_ANGOISSE.pdf).

${ }^{30} 30$ HADOT P., Exercices spirituels et philosophie antique, Paris, Albin Michel, octobre 2002, pp. 226-227.

${ }^{31} 31$ Cf. J. DARRIULAT, $(2007$ octobre $) \quad$ Introduction à $\quad$ la philosophie http://jdarriulat.net/Introductionphiloesth/http://www.jdarriulat.net/Introductionphiloesth/Antiquite/Tragediephilo/SituaTragique1.html (consulté le 12/03/2017) 